\title{
Comparison of Fracture Resistance of Different Reattachment Techniues and Adhesive Materials on Incisal Tooth Fragment Reattachment
}

\author{
Anshu Agrawal $^{1}$, Saloni Agrawal ${ }^{2}$, Dr.Deepak Kumar Sharma ${ }^{3}$, Manu bansal ${ }^{4}$, \\ Vipul Choudhary ${ }^{5}$, Kamal moolchandani ${ }^{6}$ \\ Dept Of Conservative Dentistry And Endodontics, Post Graguate ,Jaipur Dental College Rajasthan,Jaipur ${ }^{1,2}$ \\ Dept Of Conservative Dentistry And Endodontics,Jaipur Dental College , Rajasthan,Jaipur ${ }^{3,4,5,6}$
}

\begin{abstract}
Purpose: This is an in vitro study to compare fracture resistance of different reattachment techniques and adhesive materials on incisal tooth fragment reattachment.

Methodology: 100 sound human maxillary central incisors were selected .10 were maintained as a control group. Remaining 90 were divided randomly into 3 groups $(n=30)$ based upon the materials used for reattachment and further divided into 3 subgroups $(n=10)$ on the basis of different reattachment techniques. The materials used are dual cure resin cement, flowable composite, nano hybrid composite and three different reattachment techniques used were simple reattachment, overcontour and internal dentinal groove. Data was analyzed with ANOVA (One-Way) test and Post-Hoc Bonferroni test.

Results: The control group had a significantly higher fracture resistance $(p=0.001)$; the highest fracture resistance values were obtained by Dual cure resin cement followed by Nano hybrid composite and minimum in Flowable composite. When compared between three different reattachment techniques internal dentinal groove showed the maximum fracture resistance followed by over contour technique and minimum showed the simple reattachment technique.

Conclusion: Although, none of the tested materials provided fracture resistance similar to that found with the intact maxillary central incisors, utilizing the dual cure resin cement improved the fracture resistance of the reattached fragment than other materials.
\end{abstract}

Keywords: Reattachment technique; Tooth fracture; Dentin bonding agent; Composite resin

\section{Introduction}

Coronal fractures of the anterior teeth are a common form of dental trauma that mainly affects children and adolescents. ${ }^{(1,2)}$ The majority of dental injuries involves the anterior teeth, especially the maxillary incisors (because of its position in the arch), whereas the mandibular central incisors and the maxillary lateral incisors are less frequently involved ${ }^{3}$. Several factors influence the management of coronal tooth fractures, including extent of fracture (biological width violation, endodontic involvement, alveolar bone fracture), pattern of fracture and restorability of fractured tooth (associated root fracture), secondary trauma injuries (soft tissue status), presence/absence of fractured tooth fragment and its condition for use (fit between fragment and the remaining tooth structure), occlusion, esthetics, finances, and prognosis ${ }^{4-6}$. Anterior teeth trauma of a young atient is a tragic experience, which requires immediate attention not only because of damage to dentition but also because of the psychological impact it may have on the patient and parents. Various methods and techniques were employed to restore fractured teeth which include pin retained resin, orthodontic bands, stainless steel crowns, porcelain jacket crowns, and complex ceramic restorations. However all these restorations require significant tooth preparation and were not esthetically adequate; moreover they cannot be used in an emergency esthetic situation ${ }^{7-10}$.

One of the options for managing coronal tooth fractures, especially when there is no or minimal violation of the biological width, is the reattachment of the dental fragment when it is available ${ }^{11}$.Tooth fragment reattachment offers a conservative, esthetic, and cost effective restorative option that has been shown to be an acceptable alternative to the restoration of the fractured tooth with resin-based composite or fullcoverage crown ${ }^{6,12}$. Reattachment of a fragment to the fractured tooth can provide good and long-lasting esthetics (because the tooth's original anatomic form, colour, and surface texture are maintained), can restore function, can result in a positive psychological response, and is a reasonably simple procedure ${ }^{12}$. In addition, tooth fragment reattachment allows restoration of the tooth with minimal sacrifice of the remaining tooth structure. Furthermore, this technique is less time-consuming and provides a more predictable long-term wear than when direct composite is used ${ }^{11}$. Clinical trials and long-term follow-up have reported that reattachment using modern dentin bonding agents or adhesive luting systems may achieve functional and esthetic success ${ }^{13}$. 
Several aspects may govern the choice of reattachment technique. Studies have reported that the primary cause of fragment loss is new dental trauma or the non-physiological use of the restored tooth. Therefore, most concerns about reattachment techniques have been directed toward the fracture strength of the restored tooth.

Clinicians have employed an assortment of bevel designs, chamfers, dentinal and enamel grooves, and choices of resin composite materials and techniques for the reattachment of tooth fragments. Reis and colleagues ${ }^{6}$ have shown that a simple reattachment with no further preparation of the fragment or tooth was able to restore only $37.1 \%$ of the intact tooth's fracture resistance, whereas a labial chamfer recovered $60.6 \%$ of that fracture resistance; bonding with an over contour and placement of an internal groove nearly restored the intact tooth fracture strength, recovering 97.2 and $90.5 \%$ of it, respectively ${ }^{9}$. Despite the recent developments in adhesive materials and restorative techniques, there is no restorative material that can reproduce the esthetic, functional needs and the natural dental structures. Therefore, when the fractured fragment is available and ample enough to be used after dental fracture, reattachment should be considered the treatment of choice as the most conservative treatment approach. Reattachment of coronal fragments is an imperative technique for restoring fractured teeth that provide advantages over composite resin restorations or full coverage crown. It is a simple, less time consuming and low-cost method. It allows the maintenance of incisal function in dental structure and provides good and long lasting esthetics. The method maintains the natural characteristics of wear, shape, surface texture and color and produces minimal tooth loss. Consequently, it improves function and provides positive emotional response from the patient ${ }^{14}$.

\section{Materials And Methods}

A total of 100 sound human maxillary central incisors, extracted for therapeutic reasons, were used in this study. The teeth were cleaned from the soft tissues and calculus with curettes and ultrasonic devices and kept in an aqueous buffered solution of formaldehyde (Yekta Chem Co.; Tehran, Iran) for two hours to be decontaminated. The teeth were inspected under optical magnification $(\times 4)$ [16] to rule out the presence of cracks, caries or any other kind of structural defects and then stored in a sterile saline solution at room temperature until prepared for a maximum period of three months. The specimens were divided into three main groups according to adhesive material used and three subgroups according to the three different reattachment techniques used. The crown of the teeth was measured on the labial side, from the cervical line to the incisal edge and from the mesial surface to the distal surface by using a digital caliper. Each tooth was embedded in an auto polymerizing resin at cementoenamel junction. The mark line at the junction of the incisal and middle in the treatment groups was sectioned perpendicularly to the long axis of the tooth with a diamond disk using a low speed hand piece under cooling water. Thus for each tooth, one fragment was obtained. Both the fragment and the remaining tooth surface was kept in $0.9 \%$ saline solution until the restoration procedure is performed.

The treatment group was reattached as follows:

Group 1 : Control group $(n=10)$

Group 2: Material A: RELY X Unicem 3M ESPE(Dual Cure Resin Cement)

\begin{tabular}{|l|c|l|}
\hline Sub Group & $\begin{array}{c}\text { Sample } \\
\text { Size(n) }\end{array}$ & \multicolumn{1}{|c|}{ Reattachment Technique } \\
\hline $2 \mathrm{a}$ & 10 & Simple reattachment \\
\hline $2 \mathrm{~b}$ & 10 & Over Contour \\
\hline $2 \mathrm{c}$ & 10 & Internal Dentinal Groove \\
\hline
\end{tabular}

Group 3: Material B : FILTEK ${ }^{\mathrm{TM}}$ Supreme XTE (Flowable composite)

\begin{tabular}{|c|c|c|}
\hline Sub Group & $\begin{array}{c}\text { Sample } \\
\text { Size(n) }\end{array}$ & Reattachment Technique \\
\hline $3 \mathrm{a}$ & 10 & Simple reattachment \\
\hline $3 \mathrm{~b}$ & 10 & Over Contour \\
\hline 3c & 10 & Internal Dentinal Groove \\
\hline
\end{tabular}

Group 4: Material C: FILTEK Z250 XT (Nano hybrid resin based composite)

\begin{tabular}{|c|l|c|}
\hline $\begin{array}{c}\text { Sub } \\
\text { Group }\end{array}$ & $\begin{array}{c}\text { Sample } \\
\text { Size(n) }\end{array}$ & Reattachment Technique \\
\hline $4 \mathrm{a}$ & 10 & Simple reattachment \\
\hline $4 \mathrm{~b}$ & 10 & Over Contour \\
\hline $4 \mathrm{c}$ & 10 & Internal Dentinal Groove \\
\hline
\end{tabular}

\subsection{Techniques}

2.1.1 Simple reattachment $(2 \mathrm{a}, 3 \mathrm{a}, 4 \mathrm{a})$ : no additional preparation was made, fractured fragments were only bonded with three different adhesive materials.

2.1.2 Over contour $(2 \mathrm{~b}, 3 \mathrm{~b}, 4 \mathrm{~b})$ : prior to performing the reattachment of fractured tooth fragments, preparation was done on the labial surface by using a cylindrical diamond finishing bur. The preparation was extended 2.5 
mm coronally and apically from the fracture line. The increment of resin cement was used to restore the labial surface after applying the adhesive system. This created a slightly over contoured tooth surface.

2.1.3Internal dentin groove $(2 \mathrm{c}, 3 \mathrm{c}, 4 \mathrm{c})$ : prior to performing the reattachment of fractured tooth fragments, an internal dentin groove ( $1 \mathrm{~mm}$ deep and $1 \mathrm{~mm}$ wide) was placed within the fragment and the remaining tooth by using a carbide bur with a water coolant . The adhesive system was applied to each surface. Prior to light curing, a resin composite was placed within the grove. The fragment was reattached and excess was removed.

2.2 De-bonding: All the samples were thermocycled in between $\pm 5^{\circ} \mathrm{C}$ and $\pm 55^{\circ} \mathrm{C}$ for 1500 cycles at a settle time of 30s. All specimens were subjected to test in Universal Testing Machine at a cross head speed of $0.5 \mathrm{~mm} / \mathrm{min}$ in a compression mode. The block containing the reattached tooth was secured to a mounting jig at a $90^{\circ}$ angle between the load applied and a metal cone with $1.00 \mathrm{~mm}$ tip cut vertically to the center of the incisal $3^{\text {rd }}$ of the labial surface to simulate real clinical situation as possible. The maximum load to failure was recorded for each specimen and the shear bond strength was recorded in Newton(N) The data was analyzed statistically using Anova and Post-hoc Bonferroni Test. The level of significance for each test used was set to 0.05.

\section{Results}

The Anova test and Post hoc bonferroni test showed that the control group had the significantly higher fracture resistance in comparision to other groups. Based on the results of the present study the fracture resistance was highest in RELY X Unicem (Dual cure resin cement) followed by FILTEK Z250XT ( Nano hybrid composite) and lowest in FILTEK ${ }^{\mathrm{TM}}$ SUPREME XTE ( Flowable composite). When three different reattachment techniques are compared, internal dentinal groove showed the highest strength followed by over contoured and least by simple re attachment. RELY X Unicem when reattached using internal dentinal groove technique showed higher fracture resistance than over contoured technique and least by simple reattachment technique. When FILTEK Z250XT used in conjuction with internal dentinal groove reattachment technique showed highest strength followed by over contoured and simple re attachment technique and same with FILTEK $^{\mathrm{TM}}$ SUPREME XTE which showed highest fracture resistance with internal dentinal groove technique.

\begin{tabular}{|l|l|l|c|c|c|c|}
\hline S.No & Group & Mean & $\begin{array}{c}\text { Std. } \\
\text { Deviation }\end{array}$ & F-value & p-value & $\begin{array}{c}\text { Post-hoc } \\
\text { comparisons }^{\mathrm{b}}\end{array}$ \\
\hline 1. & GROUP 1 & 264.84 & 10.92 & \multirow{2}{*}{107.144} & $<0.001^{*}$ & \multirow{2}{*}{$1>2,4>3$} \\
\cline { 1 - 4 } 2. & GROUP 2 & 144.96 & 31.37 & & & \\
\cline { 1 - 3 } 3. & GROUP 3 & 107.26 & 14.29 & & & \\
\hline 4. & GROUP 4 & 133.11 & 19.05 & & & \\
\hline
\end{tabular}

Table1: The mean fracture resistance was compared between groups 1,2, 3 and 4 using the One-way ANOVA

\begin{tabular}{|c|c|c|c|c|c|c|}
\hline S.No. & $\begin{array}{l}\text { Reattachment } \\
\text { Technique }\end{array}$ & Mean & $\begin{array}{c}\text { Std. } \\
\text { Deviation }\end{array}$ & F-value & p-value ${ }^{a}$ & $\begin{array}{c}\text { Post-hoc } \\
\text { comparisons }\end{array}$ \\
\hline 1. & $\begin{array}{l}\text { Simple } \\
\text { reattachment }\end{array}$ & 98.52 & 4.47 & \multirow{3}{*}{48.916} & \multirow[t]{3}{*}{$<0.001 *$} & \multirow{3}{*}{$2,3>1$} \\
\hline 2. & Over contour & 139.42 & 27.72 & & & \\
\hline 3. & $\begin{array}{l}\text { Internal dentin } \\
\text { groove }\end{array}$ & 147.38 & 21.82 & & & \\
\hline
\end{tabular}

Table 2: The mean fracture resistance was compared between Simple reattachment, Over contour and Internal dentin groove using the One-way ANOVA test.

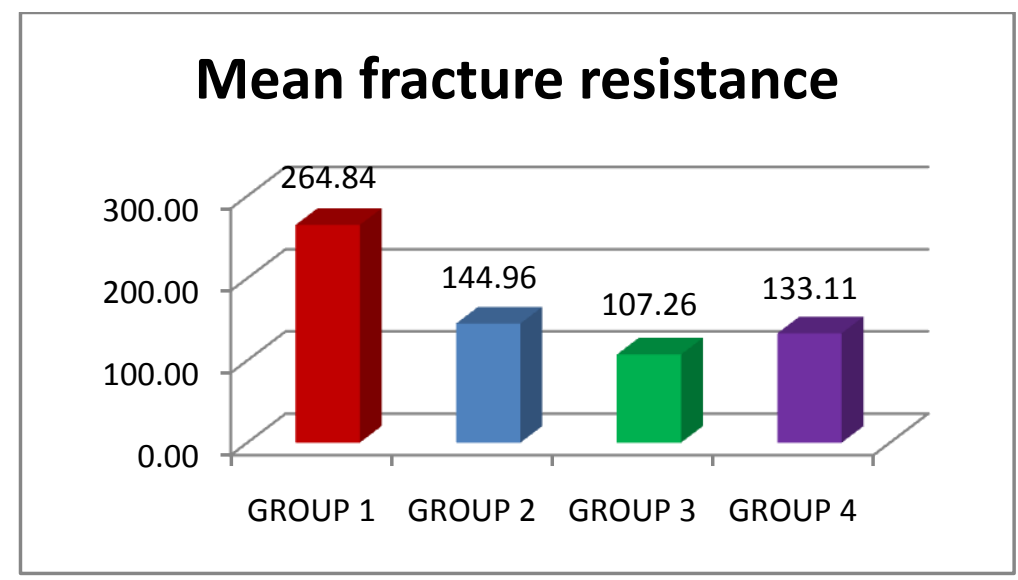

Graph 1: Graph showing mean fracture resistance of all four groups 


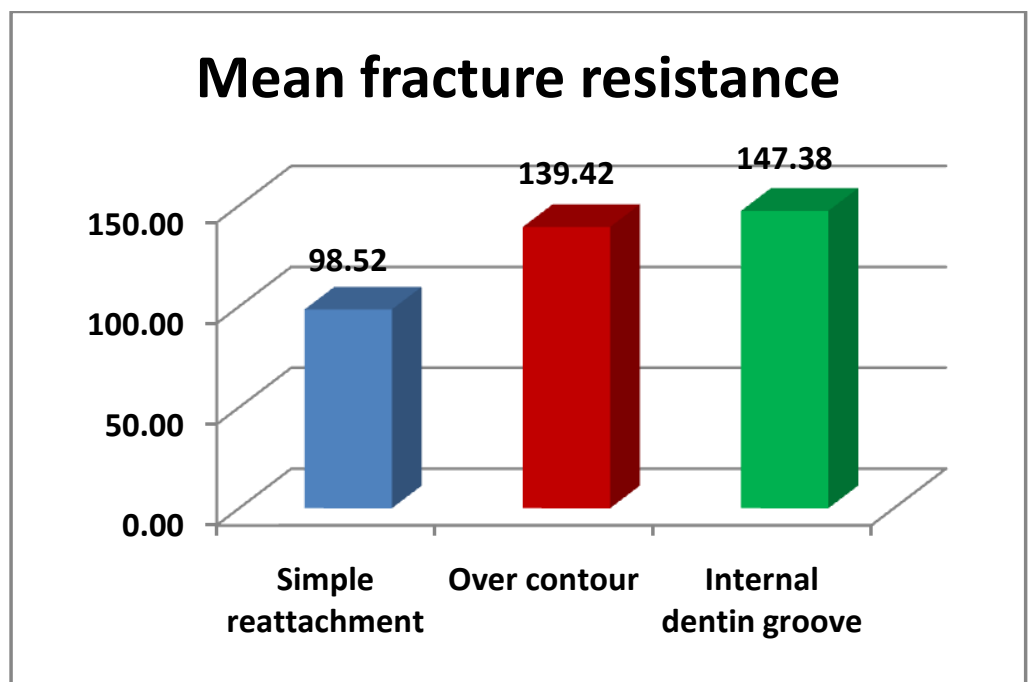

Graph 2: graph showing mean fracture resistance of three different reattachment techniques.

\section{Discussion}

Studies have shown that one out of every four persons under the age of 18 will sustain a traumatic anterior crown fracture ${ }^{15,16}$. Whenever the fracture fragment is available reattachment should be the first choice of treatment ${ }^{17}$. In recent years due to remarkable advancements of adhesive systems and resin composites, it is now possible to achieve excellent results with reattachment of tooth fragments provided that the biological factors, materials, and techniques are logically assessed and managed. In the present study, the human maxillary central incisor teeth were used to perform the tests since these teeth fracture. In addition, the specimens used for the experiment were teeth extracted for periodontal reasons, which are usually teeth of older people, whereas trauma happens usually in younger patients. Aging can cause alterations, especially in dentin, which can decrease the retention force of restorations. However, all teeth were extracted from patients of similar ages, providing a degree of homogeneity which avoids potential disparities. In this study, the incisal edge of specimens was sectioned using a diamond disk. This is the limitation of our study that the fractures differ from the natural fractures and the fragment probably will not fit well over the remaining tooth structure as precisely as in the case of a natural fracture. This situation could make it difficult to use an adhesive as a unique reattachment agent since a thicker layer of material may be necessary to fill the gaps present in the interface.

Cutting with a bur produces a smear layer which is otherwise, not present on a fractured surface. A fractured surface often runs parallel to the main direction of the enamel prisms, while the orientation of the sectioned surface is dictated by the direction of the cut. The sectioned fragment establishes standardized and repeatable condition that is absolutely necessary for an in vitro study. Also, the method employed to obtain the fragment of the incisor margin was to provoke its fracture and not to cut the crown of the teeth .

Materials used for reattachment of fractured teeth are investigated from many clinicians for they also influence strength of the connection tooth structure - fragment.. Development of contemporary composite materials and the possibilities of modern adhesive dentistry are reason for new investigations connected with reattachment technique. Andreasen FM et al. pointed out that material with comparatively high mechanical properties as composite resin should be used in combination with adhesives to outstand the functional loading. Andreasen FM et al. published in 1995 a multi centered clinical study investigating strength of reattached tooth fragments. Data come from three dental clinics both of which use only acid etching for fragment reattachment, while the third is adding a bonding agent plus acid etching. The results show that the retention level is high at fragments reattached with acid etching and bonding agent. In a contemporary study of Farik et al it is confirmed that most bonding systems fifth generation increases fracture resistance of reattached crown fragments when used in combination with resin. Self-etching adhesives have lower fracture resistance at reattachment compared to the adhesives with components in different bottles (multi bottled).

Wiegand et al., suggest the use of an internal groove when the residual dental structure and the fragment fit perfectly; otherwise an over contour is advisable when there is a partial loss of hard tissue. In this tudy we have used three adhesive materials i.e dual cure resin cement (Rely X Unicem, 3M ESPE), Flowable Composite (3M ESPE) and micro hybrid composite (Filtek Z250 3M ESPE). Dual cure resin cement showed highest fracture resistance followed by micro hybrid composite and the least is flowable composite. RelyX $\mathrm{X}^{\mathrm{TM}}$ Unicem cement is a dual-curing, self-adhesive universal resin cement for adhesive cementation of indirect ceramic, composite or metal restorations. When using Rely X Unicem cement, bonding and conditioning of the tooth are no longer necessary. The cement is characterized by a higher moisture tolerance, as compared to multistep composite cements. Rely X Unicem cement releases fluoride ions and is available in various shades. 
Among others, it's essential characteristics are high dimensional stability and very good adhesion to the tooth structure. Base paste (white) contains Methacrylate monomers containing phosphoric acid groups, Methacrylate monomers, Silanated fillers, Initiator components and Stabilizers. Catalyst paste (yellow) contains Methacrylate monomers, Alkaline (basic) fillers, Silanated fillers, Initiator components, Stabilizers and Pigments. After setting of Rely X Unicem cement, the resin matrix shows a high degree of cross-linking between the particular mono-mers. In this way good mechanical properties (e.g. high compressive and flexural strength) and adhesive bonding without pre treatment of the tooth structure can be achieved. Furthermore, a high degree of cross linking is one essential requirement for the long-term stability of the cement which is met by Rely X Unicem. Fillers have also an important impact on the cement's properties. One part of the fillers in Rely X Unicem cement is silanated and, thus, is chemically embedded into the cement matrix during setting. Another part is alkaline (basic) and thereby able to react with the phosphoric acid groups of the methacrylate monomers in a neutralization reaction. Therefore, during setting the $\mathrm{pH}$-value increases and lifts the initially acidic Rely $\mathrm{X}$ Unicem cement paste to a neutral level. This avoids hydrolysis processes in the cement in the long run and is therefore another important prerequisite for the long-term stability of any initially acidic cement. Many studies conducted showed high bond strength of Rely X Unicem when compared to different cements. Improved mechanical properties of dual cure resin cement makes the adaptation of fractured fragment better to the tooth. Its dual cure property i.e. chemical cure and light cure leads to maximum fracture resistance among all three adhesive cements.

3M ESPE Filtek ${ }^{\mathrm{TM}}$ Supreme XT Flowable Restorative is a low viscosity, visible light-cured, radiopaque flowable restorative indicated for a variety of uses including liner/base, direct restorative for Class $\mathrm{III}, \mathrm{V}$ and minimally invasive preparations, pit and fissure sealant and repair of indirect composite, porcelain and temporary acrylic materials. Filtek Supreme XT flowable restorative blends the strength and beauty of Filtek Supreme XT Universal Restorative with the "flow on demand" handling of 3M ESPE Filtek ${ }^{\mathrm{TM}}$ Flow Flowable Restorative. Using the nanofiller technology of Filtek Supreme XT universal restorative and the rheology modifier of Filtek Flow flowable restorative, 3M ESPE has developed an esthetic flowable restorative with excellent wear and polish properties and handling optimized for a variety of indications. Flowable composites, due to their limited filler loading, typically display lower physical properties than hybrid composites (Bayne 1998, Attar, 2003, Bonilla 2003). Thus, recommended indications have not included use in stress-bearing restorations. Flowable composites also typically exhibit higher polymerization shrinkage than conventional composites. Filtek ${ }^{\mathrm{TM}}$ Supreme XT Flowable Restorative is formulated with the methacrylate resin monomers Bis-GMA, TEGDMA and Bis-EMA. Filtek Supreme XT flowable restorative also contains a dimethacrylate polymer that modifies the rheology of the material and provides a "flow on demand" handling characteristic allowing the material to flow under pressure, yet hold its shape after placement until light cured. A photoinitiator component allows for light-curing when exposed to visible light in the 400-500 nanometer range.

The resin system of $3 \mathrm{M}^{\mathrm{TM}}$ Filtek ${ }^{\mathrm{TM}} \mathrm{Z} 250$ Universal Restorative consists of 3 major components. In Filtek Z250 restorative, the majority of TEGDMA has been replaced with a blend of UDMA (urethane dimethacrylate) and Bis-EMA(6)1 (Bisphenol A polyetheylene glycol dietherdimethacrylate). Both of these resins are of higher molecular weight and therefore have fewer double bonds per unit of weight. The high molecular weight materials also impact the measurable viscosity. A typical batch of $3 \mathrm{M}^{\mathrm{TM}} \mathrm{Z100}{ }^{\mathrm{TM}}$ Restorative has a viscosity of 30,000 poise, whereas Filtek Z250 universal restorative has a target viscosity of 350,000 poise. Despite this large disparity, dentists may not distinguish any difference in handling viscosity. However, the higher molecular weight of the resin results in less shrinkage, reduced aging and a slightly softer resin matrix. Additionally these resins impart a greater hydrophobicity and are less sensitive to changes in atmospheric moisture. The particle size distribution is $0.01 \mu \mathrm{m}$ to $3.5 \mu \mathrm{m}$ with an average particle size of $0.6 \mu \mathrm{m}$.

Various clinicians have used a different bevel designs, chamfers, dentinal and enamel grooves for the reattachment of tooth fragments ${ }^{20}$. The techniques used in this study are simple reattachment technique, over contour and internal dentinal groove. In the study internal dentinal groove showed the highest fracture resistance which was statistically significant followed by over contour and simple reattachment showing the least fracture resistance. Placement of internal dentinal groove may provide excellent fracture strength and highest esthetic durability. It is likely that the greater adhesion area and permeability of internal resin bar which acts as an opponent to the compression load applied on the buccal surface could be responsible for the better results obtained in this group. The cement goes inside the internal groove given on the tooth as well as the fractured fragment and bears the load.

In overcontouring (group $2 b, 3 b, 4 b$ ) good performance may be attributed to enlargement of adhesion area provided by tooth preparation around the fracture site. The greater extension of material on the surface, the better force distribution over a large surface area,contrary to what occurred in simple reattachment, where the stress concentration is in the fracture line. However this greater exposure of composite resin may diminish the long term esthetics due to the process of abrasion and discoloration that occurs due to composite with time. Polishing at regular intervals may solve the problem. 
The simple reattachment (group 2a,3a,4a) had an overall fracture strength recovery of $36.6 \%$, which indicated that reattachment without preparation exhibited less than $50 \%$ of fracture strength displayed by intact teeth. A study was conducted by Yilmaz et al in 2008 to evaluate clinically and radiographically the restored teeth using reattachment technique of fractured fragment to the remaining tooth with flowable resin composite. The restored teeth were accesed in terms of parental-patient ratings of satisfaction. Both clinically and radiographically no pathology was reported and all the restorations were successful.However, considering the proposed advantages of these techniques, materials and their widespread use, the majority of the design features are selected empirically and little is known about their influence in long term success of restorations. Hence these variables necessitate future critical evaluation.

\section{Conclusion}

Within the limited parameters of this study, the following conclusion can be drawn:

- Dual cure resin cement (RELY X Unicem) shows the highest fracture resistance in comparison to nano hybrid composite (FILTEK Z250 XT) and flowable composite (FILTEK ${ }^{\mathrm{TM}}$ Supreme XTE).

- Adhesive material incorporated for reattachment gives it a better strength in comparison to reattachment using only bonding agent.

- Different preparations showed better fracture resistance in comparison to simple reattachment technique i.e. without any preparation for reattachment.

- In comparison between internal dentinal groove, over contour and simple reattachment internal dentinal groove shows the highest fracture resistance.

References
[1]. Dietschi D, Jacoby T, Dietschi JM, Schatz JP. Treatment of traumatic injuries in the front teeth: restorative aspects in crown fractures. Pract Periodontics Aesthetic Dent PPAD. 2000 Oct;12(8):751-758; quiz 760.

[2]. Hamilton FA, Hill FJ, Holloway PJ. An investigation of dento-alveolar trauma and its treatment in an adolescent population. Part 1: The prevalence and incidence of injuries and the extent and adequacy of treatment received. Br Dent J. 1997 Feb 8;182(3):91-5.

[3]. Andreasen JO, Andreasen FM, L. Andersson. Textbook and Color Atlas of Traumatic Injuries of the Teeth. 4th ed.

[4]. Andreasen FM, Norén JG, Andreasen JO, Engelhardtsen S, Lindh-Strömberg U. Long-term survival of fragment bonding in the treatment of fractured crowns: a multicenter clinical study. Quintessence Int Berl Ger 1985. 1995 Oct;26(10):669-81.

[5]. Olsburgh S, Jacoby T, Krejci I. Crown fractures in the permanent dentition: pulpal and restorative considerations. Dent Traumatol Off Publ Int Assoc Dent Traumatol. 2002 Jun;18(3):103-15.

[6]. Reis A, Francci C, Loguercio AD, Carrilho MR, Rodriques Filho LE. Re-attachment of anterior fractured teeth: fracture strength using different techniques. Oper Dent. 2001 Jun;26(3):287-94.

[7]. Badami AA, Dunne SM, Scheer B. An in vitro investigation into the shear bond strengths of two dentine-bonding agents used in the reattachment of incisal edge fragments. Endod Dent Traumatol. 1995 Jun;11(3):129-35.

[8]. Buonocore MG, Davila J. Restoration of Fractured Anterior Teeth with Ultraviolet-Light-Polymerized Bonding Materials: A New Technique. J Am Dent Assoc. 1973 Jun 1;86(6):1349-54.

[9]. Andreasen JO. Buonocore memorial lecture. Adhesive dentistry applied to the treatment of traumatic dental injuries. Oper Dent. 2001 Aug;26(4):328-35.

[10]. Goenka P, Dutta S, Marwah N. Biological approach for management of anterior tooth trauma: triple case report. J Indian Soc Pedod Prev Dent. 2011 Jun;29(2):180-6.

[11]. Baratieri LN, Monteiro S, Caldeira de Andrada MA. Tooth fracture reattachment: case reports. Quintessence Int Berl Ger 1985. 1990 Apr;21(4):261-70.

[12]. Maia EAV, Baratieri LN, de Andrada MAC, Monteiro S, de Araújo EM. Tooth fragment reattachment: fundamentals of the technique and two case reports. Quintessence Int Berl Ger 1985. 2003 Feb;34(2):99-107.

[13]. Oz IA, Haytaç MC, Toroglu MS. Multidisciplinary approach to the rehabilitation of a crown-root fracture with original fragment for immediate esthetics: a case report with 4-year follow-up. Dent Traumatol Off Publ Int Assoc Dent Traumatol. 2006 Feb;22(1):4852.

[14]. Demarco FF, Fay R-M, Pinzon LM, Powers JM. Fracture resistance of re-attached coronal fragments--influence of different adhesive materials and bevel preparation. Dent Traumatol Off Publ Int Assoc Dent Traumatol. 2004 Jun;20(3):157-63.

[15]. J. O. Andreasen and J. J. Ravn, "Epidemiology of traumatic dental injuries to primary and permanent teeth in a Danish population sample," International Journal of Oral Surgery, vol.1, no. 5, pp. 235-239, 1972.

[16]. S. Petti and G. Tarsitani, "Traumatic injuries to anterior teeth in Italian schoolchildren: prevalence and risk factors," Endodontics and Dental Traumatology, vol. 12, no. 6, pp. 294-297, 1996.

[17]. Y. Yilmaz, C. Zehir, O. Eyuboglu, and N. Belduz, "Evaluation of success in the reattachment of coronal fractures," Dental Traumatology, vol. 24, no. 2, pp. 151-158, 2008

[18]. Liebenberg WH. Reattachment of a coronal fragment: operative considerations. Pract Periodont AesthDent 1997; 9: 761-772.

[19]. Simonsen RJ. Restoration of a fractured central incisor using original tooth fragment.J Am Dent Assoc. 1982 Oct;105(4):646-8.

[20]. Borssen E, Holm AK. Traumatic dental injuries in a cohort of 16-year-olds in northern Sweden .Endod Dent Traumatol 1997; 13: 276-280 